\title{
Matrix Methods for Optimal Manifesting of Multi-Node Space Exploration Systems
}

\author{
Paul T. Grogan ${ }^{1}$, Afreen Siddiqi ${ }^{2}$, and Olivier L. de Weck ${ }^{3}$ \\ Massachusetts Institute of Technology, Cambridge, MA, 02139
}

\begin{abstract}
This paper presents matrix-based methods for determining optimal cargo manifests for space exploration. An exploration system is defined as a sequence of in-space and on-surface transports between multiple nodes coupled with demands for resources. The goal is to maximize value and robustness of exploration while satisfying logistical demands and physical constraints at all times. To reduce problem complexity, demands are abstracted to a single class of resources and one metric (e.g. mass or volume) governs capacity limits. Matrices represent cargo carried by transports, cargo used to satisfy demands, and cargo transferred to other transports. A system of equations enforces flow conservation, demand satisfaction, and capacity constraints. Exploration system feasibility is evaluated by determining if a solution exists to a linear program or network flow problem. Manifests are optimized subject to an objective function using linear or non-linear programming techniques. In addition to modeling the manifesting problem, a few metrics such as the transport criticality index are formulated to enable analysis and interpretation. The proposed matrix manifest modeling methods are demonstrated with a notional lunar exploration system comprised of 32 transports including 8 cargo and 9 crewed landings at an outpost at the Lunar South Pole and several surface excursions to Malapert Crater and Schrodinger Basin. It is found that carry-along and pre-positioning logistics strategies yield different manifesting solutions in which transport criticality varies. For the lunar scenario, transport criticality is larger for a pre-positioning strategy (mean value of 3.02) as compared to an alternative carry-along case (mean-value of 1.99).
\end{abstract}

\footnotetext{
${ }^{1}$ Graduate Research Assistant, Department of Aeronautics and Astronautics, 33-409, AIAA Student Member.

${ }^{2}$ Research Scientist, Engineering Systems Division, E40-231, AIAA Member.

${ }^{3}$ Associate Professor, Department of Aeronautics and Astronautics, 33-410, AIAA Associate Fellow.
} 


\section{Nomenclature}

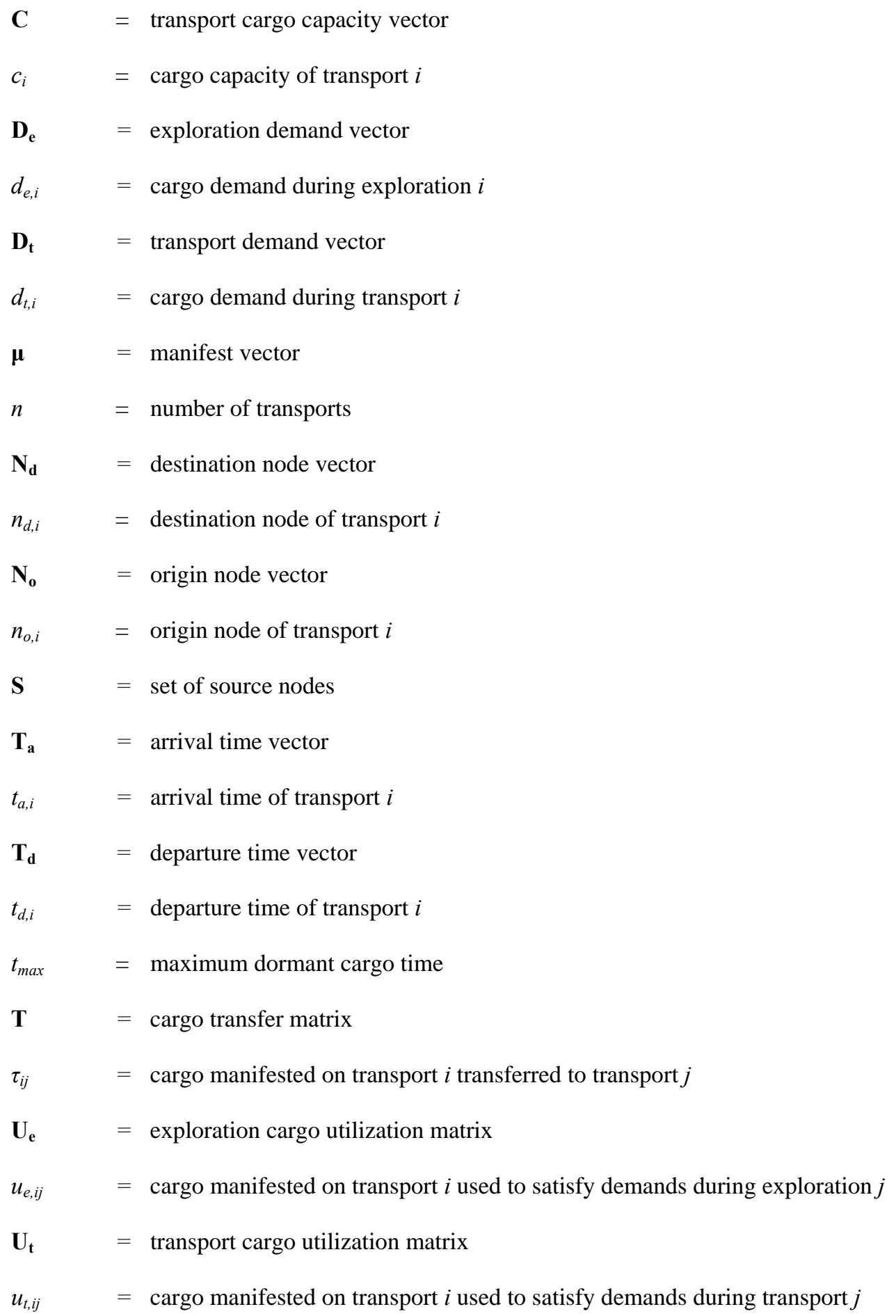




\section{Introduction}

MANIFESTING resources and cargo onto launch vehicles and in-space transports is an integral part of planning space exploration. Past and present space missions reduced manifest complexity by limiting interdependencies between transports. The Apollo program used a sortie approach where all required cargo was carried on a single launch for each mission. In contrast, the International Space Station receives deliveries from many launches but only requires manifesting decisions across one transportation link, i.e. there is no intermediate transfer of cargo. These sortie and single-chain methods of manifesting will need to be expanded as humans seek to establish long-duration research stations and explore distant locations including near-Earth objects, Lagrange Points and Mars. Future space exploration may depend upon on-orbit construction, refueling and depots, surface caching, and a host of other advanced logistics strategies. Furthermore, a well-planned manifesting strategy based on pre-positioned, carryalong, and re-supply cargo is essential for balancing risks, ensuring robustness against delays and cancellations, and achieving maximum exploration capability and success [1]. As a step towards this goal, this paper illustrates how a cargo manifest for extended exploration missions and campaigns can be obtained and optimized through relatively low computational effort such that the important feasibility constraints are met while maintaining tractability for integration into strategic analyses.

The manifesting problem is not limited to exploration in space. In 1804 Lewis and Clark set out on an extensive surface exploration reaching across the newly-acquired American West from St. Louis to the Pacific Ocean. Prior to departure the expedition included extensive planning for trading with Native Americans, selection of wintering locations, and caching of goods to retrieve on the return voyage [2]. A century later, expeditions to the South Pole led by Scott, Shackleton, and Amundsen reinforced the importance of advance planning and depot establishment to survive the harsh Antarctic environment for months at a time [3]. Largely unaided by mathematical models, historical exploration relied on the expertise of leaders coupled with a bit of luck for success and "living off the land.” Modern terrestrial exploration embraces the use of airborne operations to significantly decrease travel time and provide resupply flexibility. "Food may also be sent in advance, but if distribution centres are within [helicopter] range it is more satisfactory to service the camp from these centres, thus providing fresh food and mail service” writes Blackadar on arctic exploration in the 1950s [4]. 
Modern scientific approaches to logistics planning are prevalent in the military and business industry, broadly covered by the field of operations research. Rapid developments in computation have enabled the analysis of largescale problems using mathematical models. Military logistics models focus on the routing and scheduling of vehicles and distribution of supplies from depots to combat units [5]. Baker et al. for example developed a largescale linear programming model to optimize routing of people and cargo using several types of aircraft between multiple nodes [6]. Within business applications, logistics models evaluate the frequency, capacity, route, and sequence of transports as well as select depot and warehouse locations based on competition (quality, delivery time, etc.) and cost factors [7]. Klingman et al. for example developed a large-scale linear programming model to optimize the production and distribution of phosphate-based chemical products [8].

There are three major differences between space exploration systems and their modern terrestrial counterparts. First, space exploration operates on a long timescale. Exploring outside the Earth-moon system requires significantly longer durations (weeks and months) compared to terrestrial transport operations which tend to unfold in hours and days, with the exception of ocean freight. The long-duration transports also contribute to in-flight demands which may even exceed the demands at the destination. Second, space transportation schedules are highly constrained. Whereas on Earth scheduling transportation is generally flexible, space transports are limited by the astrodynamics of planetary motion and long lead time of spacecraft fabrication and ground operations. The schedule rigidity emphasizes the importance of advanced planning and robustness to uncertainty. Finally, space transportation cargo is extraordinarily critical. As there are no known resources in space or on planetary bodies to support life in raw form and in-situ processing capabilities rely on additional systems, even a slight undersupply corresponding to a resource or component could lead to serious failures. Furthermore, usable cargo comprises only $0.5 \%-1.5 \%$ of a launch vehicle's wet mass to low-Earth orbit compared to nearly $50 \%$ of a truck's gross weight and $10 \%$ of an airplane’s gross takeoff weight. These differences - timescale, schedule, and criticality - emphasize different aspects of the manifesting model for space exploration than that of terrestrial logistics operations.

Previous work established matrix-based methods for modeling the manifesting process which was demonstrated with an analysis of the International Space Station resupply logistics and a lunar surface exploration campaign [9]. It was shown how the cargo manifests for a multi-flight, single-node scenario can be represented with a square, M 
(manifest)-matrix. An in-depth discussion was provided on the structure of the M-matrix, its implications for campaign logistics, and its use for deriving various mission- and campaign-level logistics metrics.

The existing methods have been further expanded upon using a modeling framework developed for integrated space logistics simulation to allow for more general multi-transport, multi-node scenarios [10]. The expanded framework presented in this paper provides matrix-based methods for a wider range of scenarios under consideration for future exploration campaigns including long-duration crewed missions, necessary for any exploration beyond low-Earth orbit.

\section{Matrix Modeling Framework}

Matrices are a convenient notation for use in modeling frameworks as they are compact and easily accessed within optimization functions. This section describes how both space exploration systems and cargo manifests can be represented in matrix form.

\section{A. Modeling Space Exploration Systems}

Previous work introduced matrix methods for modeling flight manifests using the assumption that there are several crewed and un-crewed flights carrying cargo to a specific location, or node [9]. Though past work used the concept of flights to deliver supplies, a more general modeling framework uses the notion of a transport, previously applied in the context of integrated space exploration logistics [10]. A transport is defined as the discrete movement of cargo up to a capacity limit from an origin node to a destination node with associated departure and arrival times. A transport may be a space flight (such as between the Earth and the Moon), or a surface traversal (such as between two craters on the Moon). The capacity limit represents the remaining capacity for cargo after considering any premanifested elements such as crew, science payloads, infrastructure, etc. and is typically expressed in either mass or volume units depending on the limiting constraint of the transportation system and analysis fidelity.

A space exploration system is a series of transports coupled with demands for cargo over time. Demands have both a temporal and spatial dimension, and cargo is assumed to be available for consumption during transport or immediately upon arrival and is shared by all co-located elements. In this simplified model, demands are limited to a single generic cargo class combining any underlying resources and packaging or overhead into one quantity to analyze the manifest in aggregate. Consumption of cargo means resources are utilized (removed from the scope of 
the model) to satisfy demands with no waste, empty packaging, or other byproducts generated. Though simplifying, these assumptions do not greatly affect the usefulness of the manifest model at a strategic level of analysis. First, in looking at a single transport capacity metric (e.g. mass or volume), all cargo is equivalent independent of function and finer-level detail is not necessary to create a manifest. Second, useless waste or byproducts generated during consumption would likely be discarded or jettisoned to avoid unnecessary use of cargo capacity. Finally, higherlevel processes such as in-situ resource utilization (ISRU) or environmental and life support system (ECLSS) processes including water recovery which depend on the transformation of resources (possibly byproducts of consumption) can be modeled outside the scope of the manifesting model using an integrated demand simulator. The resulting demand quantities therefore serve as an input to the manifesting model.

Demands for cargo occurring at nodes are aggregated by exploration period, defined as the interval between subsequent arrivals of transports or the end of the campaign. There exists exactly one exploration period for each transport, though each period need not include demands. Demand aggregation simplifies the model without loss of generality as no cargo could be delivered in the middle of an exploration period by definition. Similarly, demands for cargo that occur while in transit are aggregated to the start of the respective transport. Figure 1 illustrates a timeexpanded network (bat chart) for a sample dual-launch lunar sortie. Kennedy Space Center (KSC) is the origin node from which two transports $T_{1}$ and $T_{2}$ emanate to deliver cargo to low Earth orbit (LEO). Two exploration periods $E_{1}$ and $\mathrm{E}_{2}$ occur at LEO before transport $\mathrm{T}_{3}$ departs for low lunar polar orbit (LLPO). Immediately upon arrival of $\mathrm{T}_{3}$ exploration period $E_{3}$ occurs followed by transport $T_{4}$ to the ultimate destination, the Lunar South Pole (LSP). The return to the Pacific splashdown zone (PSZ) uses transports $\mathrm{T}_{5}$ and $\mathrm{T}_{6}$ via LLPO.

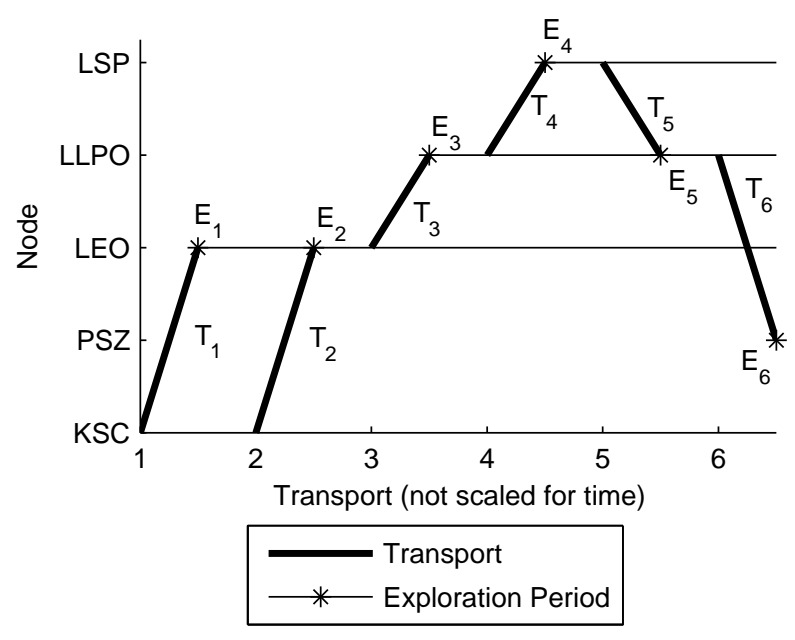

6

American Institute of Aeronautics and Astronautics 
Fig. 1 Example bat chart showing transports and exploration periods.

Given this terminology, a space exploration system is completely defined in matrix format with seven components: the origin node vector $\mathbf{N}_{\mathbf{o}}$, the destination node vector $\mathbf{N}_{\mathbf{d}}$, the departure time vector $\mathbf{T}_{\mathbf{d}}$, the arrival time vector $\mathbf{T}_{\mathbf{a}}$, the transport capacity vector $\mathbf{C}$, the exploration demands vector $\mathbf{D}_{\mathbf{e}}$, and the transport demands vector $\mathbf{D}_{\mathbf{t}}$, as shown in Eqs. (1-7) for a campaign of $n$ transports. Table 1 defines the dual-launch lunar sortie exploration system in terms of these seven vectors.

$$
\begin{aligned}
\mathbf{N}_{\mathbf{o}} & =\left[\begin{array}{llll}
n_{o, 1} & n_{o, 2} & \cdots & n_{o, n}
\end{array}\right]^{T} \\
\mathbf{N}_{\mathbf{d}} & =\left[\begin{array}{llll}
n_{d, 1} & n_{d, 2} & \cdots & n_{d, n}
\end{array}\right]^{T} \\
\mathbf{T}_{\mathbf{d}} & =\left[\begin{array}{llll}
t_{d, 1} & t_{d, 2} & \cdots & t_{d, n}
\end{array}\right]^{T} \\
\mathbf{T}_{\mathbf{a}} & =\left[\begin{array}{llll}
t_{a, 1} & t_{a, 2} & \cdots & t_{a, n}
\end{array}\right]^{T} \\
\mathbf{C} & =\left[\begin{array}{llll}
c_{1} & c_{2} & \cdots & c_{n}
\end{array}\right]^{T} \\
\mathbf{D}_{\mathbf{e}} & =\left[\begin{array}{llll}
d_{e, 1} & d_{e, 2} & \cdots & d_{e, n}
\end{array}\right]^{T} \\
\mathbf{D}_{\mathbf{t}} & =\left[\begin{array}{llll}
d_{t, 1} & d_{t, 2} & \cdots & d_{t, n}
\end{array}\right]^{T}
\end{aligned}
$$

Table 1 Dual-launch sortie exploration system definition

\begin{tabular}{rcrcrrrr}
\hline \hline $\boldsymbol{i}$ & $\boldsymbol{n}_{\boldsymbol{o}, \boldsymbol{i}}$ & $\begin{array}{c}\boldsymbol{t}_{\boldsymbol{d}, \boldsymbol{i}} \\
{[\mathrm{day}]}\end{array}$ & $\boldsymbol{n}_{\boldsymbol{d}, \boldsymbol{i}}$ & $\begin{array}{c}\boldsymbol{t}_{\boldsymbol{a}, \boldsymbol{i}} \\
{[\mathrm{day}]}\end{array}$ & $\begin{array}{c}\boldsymbol{c}_{\boldsymbol{i}} \\
{[\mathrm{kg}]}\end{array}$ & $\begin{array}{c}\boldsymbol{d}_{\boldsymbol{t}, \boldsymbol{i}} \\
{[\mathrm{kg}]}\end{array}$ & $\begin{array}{c}\boldsymbol{d}_{\boldsymbol{e}, \boldsymbol{i}} \\
{[\mathrm{kg}]}\end{array}$ \\
\hline 1 & KSC & 0 & LEO & 1 & 100 & 25 & 25 \\
2 & KSC & 2 & LEO & 3 & 500 & 0 & 25 \\
3 & LEO & 4 & LLPO & 7 & 600 & 75 & 25 \\
4 & LLPO & 8 & LSP & 9 & 300 & 25 & 250 \\
5 & LSP & 14 & LLPO & 15 & 50 & 25 & 25 \\
6 & LLPO & 16 & PSZ & 20 & 300 & 100 & 0 \\
\hline \hline
\end{tabular}


The process of manifesting allocates cargo onto transports attempting to satisfy all demands without violating temporal or capacity constraints within the space exploration system, rendering it feasible from a logistics perspective. Propulsive feasibility is assumed to exist even if all flight capacities, $\mathbf{C}$, are fully utilized.

\section{B. Modeling Cargo Manifests}

A space exploration system manifest is broken down into three components defining where and how cargo is used, illustrated in Fig. 2 for manifesting actions between transports $i$ and $j$. Elements of the exploration utilization matrix $\mathbf{U}_{\mathfrak{e}}$, and transport utilization matrix $\mathbf{U}_{\mathfrak{t}}$, indicate cargo to be consumed to satisfy demands during exploration and transport, respectively. Elements from the transfer matrix $\mathbf{T}$ indicate cargo to be transferred from one transport to another for future utilization.

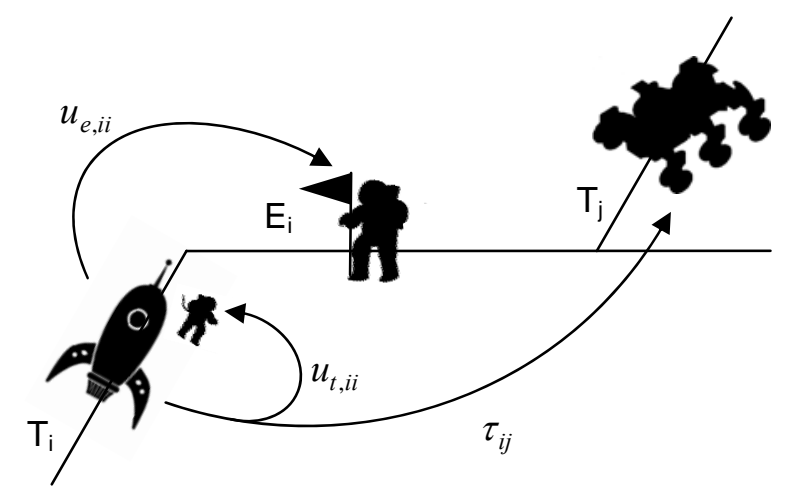

Fig. 2 Valid manifesting actions between transports $i$ and $j$.

The element $u_{e, i j}$, as shown in Eq. (8) for a campaign of $n$ transports, represents cargo brought by transport $i$ that is consumed in exploration period $j$ of the campaign. Cargo utilization between transports is only valid if both transports have the same destination node. Terms in the upper-triangular portion of the exploration utilization matrix represent pre-positioned cargo (delivered before it was demanded), and terms along the diagonal represent carryalong cargo (delivered as it was demanded). If a demand cannot be satisfied during the exploration period in which it is needed, a future transport may satisfy the demand via a backorder (delivered after it was demanded). Backorders are represented in the lower-triangular portion of the exploration utilization matrix. 


$$
\mathbf{U}_{\mathbf{e}}=\left[\begin{array}{cccc}
u_{e, 11} & u_{e, 12} & \cdots & u_{e, 1 n} \\
u_{e, 21} & u_{e, 22} & \cdots & u_{e, 2 n} \\
\vdots & \vdots & \ddots & \vdots \\
u_{e, n 1} & u_{e, n 2} & \cdots & u_{e, n n}
\end{array}\right]
$$

Crewed missions, especially ones with long-duration transports, are highly dependent on demands experienced during transit. These demands are different from exploration demands because they must be satisfied by the transport in which they originate, i.e. pre-positioning is not allowed. The element $u_{t, i j}$, as shown in Eq. (9) for a campaign of $n$ transports, represents cargo brought by transport $i$ that is consumed during transport $j$. Transport utilization typically will only occur within a single transport, represented along the diagonal of the transport utilization matrix. If allowed, backorders are valid only if both transports must have the same destination node and are represented in the upper- or lower-triangular portions of the matrix.

$$
\mathbf{U}_{\mathbf{t}}=\left[\begin{array}{cccc}
u_{t, 11} & u_{t, 21} & \cdots & u_{t, 1 n} \\
u_{t, 12} & u_{t, 22} & \cdots & u_{t, 2 n} \\
\vdots & \vdots & \ddots & \vdots \\
u_{t, n 1} & u_{t, n 2} & \cdots & u_{t, n n}
\end{array}\right]
$$

The element $\tau_{i j}$, as shown in Eq. (10) for a campaign of $n$ transports, represents cargo brought by transport $i$ that is transferred to transport $j$. Transfer of cargo does not necessarily indicate physical movement if the same underlying vehicles are used in both transports. A transfer is valid only if the destination node of transport $i$ is the origin node of transport $j$ and the arrival time of transport $i$ is before the departure time of transport $j$. As a result of these constraints, transfer terms will only occur in the upper-triangular portion of the transfer matrix.

$$
\mathbf{T}=\left[\begin{array}{cccc}
\tau_{11} & \tau_{12} & \cdots & \tau_{1 n} \\
\tau_{21} & \tau_{22} & \cdots & \tau_{2 n} \\
\vdots & \vdots & \ddots & \vdots \\
\tau_{n 1} & \tau_{n 2} & \cdots & \tau_{n n}
\end{array}\right]
$$

The resulting manifest $\boldsymbol{\mu}$ is the vector defined by the set of inputs that drive the movement of cargo throughout a campaign, given by the valid elements of the matrices $\mathbf{U}_{\mathfrak{e}}, \mathbf{U}_{\mathfrak{t}}$, and $\mathbf{T}$. The upper-bound manifest vector length is $3 \cdot n^{2}$ 9 American Institute of Aeronautics and Astronautics 
where $n$ is the number of transports in the space exploration system. In practice, however, most of the elements are excluded from consideration due to four spatial and temporal validity conditions restricting possible manifest actions, shown in Eq. (11).

$$
\boldsymbol{\mu} \equiv\left\{\mathbf{U}_{\mathbf{e}}, \mathbf{U}_{\mathbf{t}}, \mathbf{T}\right\} \forall\left\{\begin{array}{c}
u_{e, i j} \mid n_{d, i}=n_{d, j} \\
u_{t, i j} \mid n_{d, i}=n_{d, j} \\
\tau_{i j} \mid n_{d, i}=n_{o, j} \\
\tau_{i j} \mid t_{a, i} \leq t_{d, j}
\end{array}\right.
$$

Furthermore, if backorders are not allowed two additional conditions further restrict the supply of cargo for exploration and transport utilization. The transport utilization condition supersedes the previous constraint, resulting in five total constraints as shown in Eq. (12). The constraints require that exploration utilization is allowed for transports having identical destinations and arrivals before the demands are needed (constraints 1 and 2), transportation utilization is only allowed within a transport (constraint 3), and resource transfer is allowed for transports arriving and departing from identical nodes where the arrival occurs before the departure (constraints 4 and 5). Manifest vectors for a system with $n$ transports and no backorders range from length $3 \cdot n$ for a purely sequential architecture to $\left(n^{2}+3 \cdot n\right) / 2$ for a single-destination architecture.

$$
\boldsymbol{\mu} \equiv\left\{\mathbf{U}_{\mathbf{e}}, \mathbf{U}_{\mathbf{t}}, \mathbf{T}\right\} \forall\left\{\begin{array}{l}
u_{e, i j} \mid n_{d, i}=n_{d, j} \\
u_{e, i j} \mid t_{a, i} \leq t_{a, j} \\
u_{t, i j} \mid i=j \\
\tau_{i j} \mid n_{d, i}=n_{o, j} \\
\tau_{i j} \mid t_{a, i} \leq t_{d, j}
\end{array}\right.
$$

\section{Exploration System Feasibility Criteria}

A feasible space exploration system must satisfy three criteria: capacity constraints, demand satisfaction, and flow conservation. Capacity constraints ensure the sum of exploration utilization, transport utilization, and transferred cargo does not exceed the capacity of each transport, as shown in Eq. (13).

$$
\sum_{j}\left(u_{e, i j}+u_{t, i j}+\tau_{i j}\right) \leq c_{i} \forall i
$$


Demand satisfaction ensures that cargo is utilized to satisfy all exploration and transportation demands, as shown in Eqs. (14-15).

$$
\begin{aligned}
& \sum_{j} u_{e, j i}=d_{e, i} \forall i \\
& \sum_{j} u_{t, j i}=d_{t, i} \forall i
\end{aligned}
$$

Flow conservation ensures that cargo is not created or destroyed outside of demand utilization within the exploration system (again, detailed ISRU and ECLSS operations are not modeled at the manifest level, these effects can be represented in the cargo demands). For each transport, the total cargo transferred in must equal the sum of cargo transferred out plus any exploration or transport demands utilized. To enable the instantiation of cargo at special source nodes (e.g. launch sites), any transports that originate from a set of nodes $\mathbf{S}$ are exempted. The resulting constraint is shown in Eq. (16).

$$
\sum_{j}\left(\tau_{j i}-u_{e, i j}-u_{t, i j}-\tau_{i j}\right)=0 \forall i \mid n_{o, i} \notin \mathbf{S}
$$

The space exploration system is considered feasible if a solution exists to a system of linear inequality equations based on the above constraints, summarized in Eq. (17). The A-matrices impose constraints on the manifest vector elements. $\mathbf{A}_{\mathbf{c}}$ imposes capacity constraints from Eq. (13), $\mathbf{A}_{\mathbf{d e}}$ imposes exploration demand constraints from Eq. (14), $\mathbf{A}_{\mathrm{dt}}$ imposes transport demand constraints from Eq. (15), and $\mathbf{A}_{\mathbf{f}}$ imposes flow conservation constraints from Eq. (16). Finally, as the model only operates forward in time, manifest components cannot be negative.

$$
\text { find } \boldsymbol{\mu} \text { such that }\left\{\begin{aligned}
\mathbf{A}_{\mathrm{c}} \cdot \boldsymbol{\mu} & \leq \mathbf{C} \\
\mathbf{A}_{\mathrm{de}} \cdot \boldsymbol{\mu} & =\mathbf{D}_{\mathbf{e}} \\
\mathbf{A}_{\mathrm{dt}} \cdot \boldsymbol{\mu} & =\mathbf{D}_{\mathbf{t}} \\
\mathbf{A}_{\mathrm{f}} \cdot \boldsymbol{\mu} & =0 \\
\boldsymbol{\mu} & \geq 0
\end{aligned}\right.
$$


By expressing the manifesting problem as a system of inequalities, linear programming (LP) methods can be used to find a solution and determine over-all exploration feasibility. Under any objective function $J$, if the system of inequalities produces a solution, the exploration system is feasible. A simple but useful objective function is

$J(\boldsymbol{\mu})=\sum_{i} \mu_{i}$ which finds a minimum flow manifest, avoiding needless circulation of cargo. In most feasible systems, a minimum flow manifest is not unique and specific solutions may be obtained using other objective functions or additional constraints. In the event that a space exploration system is infeasible, the constraints may be relaxed, e.g. backorders may be allowed. If the modified exploration system is feasible, its Lagrange multipliers may be used to identify the most active constraints as suggestions for system modifications.

\section{Flow Network Graph Formulation}

The manifesting problem as described and formulated in Section C essentially models the movement of cargo in a flow network. A time-expanded flow network enables analysis by reformulating the dynamic network as a series of static networks at each time step [11-13]. The extensive literature and tools of graph theory can then be applied to obtain insights into the exploration system problem, including new methods to establish feasibility and visual companions to matrices.

To model as a time-expanded flow network graph, each transport is represented by a composition of four network flow nodes; two designate the departure and arrival points of the transport while two notate the exploration and transportation demand sinks. Source transports replace the departure node with a source node. Arcs connect the nodes of one transport to others to represent valid cargo movement. In Fig. 3 we see the basic building block of a space exploration system represented as a glyph (a generic network component) with several flows entering and leaving the control volume: (a) utilized pre-positioned cargo, (b) transfer cargo received, (c) dispatched transfer cargo, and (d) future pre-positioned cargo. 


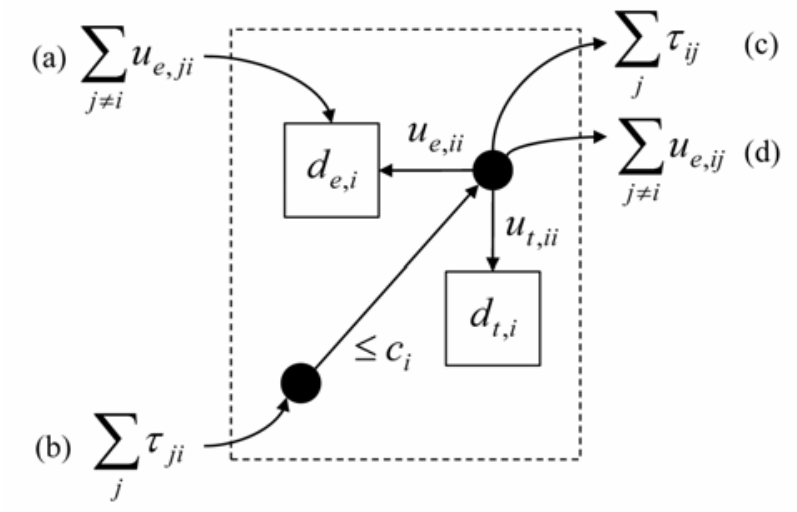

Fig. 3 Flow network glyph for transport $i$.

The space exploration system can be formulated as a minimum cost flow problem [11]. This type of flow network requires a unit cost and maximum capacity on each arc and a supply or demand associated with each node. In the space exploration system case, only the transportation arc between the departure and arrival node is bounded by the transport's capacity; all other arcs are unbounded. A minimum flow manifest can be found if every arc is assigned the same, non-zero unit cost. Also, all source nodes are connected to a virtual "super source” node having supply equal to the sum of all transportation and exploration demands and all sink nodes retain respective demands locally. The "super source" node represents in effect the space enterprise supply chain - currently only available on Earth that is the network of suppliers that creates the cargo items that are then subsequently transported and consumed during space exploration. In the long-term future such "super source" nodes could potentially also exist on the Moon, on Mars and at other locations should the footprint of human civilization expand in this way.

Figure 4 illustrates the combination of transportation glyphs to construct a complete space exploration system flow network graph corresponding to the dual-launch lunar sortie defined in Table 1. The "super source" node supplying the two source (white) nodes representing the Kennedy Space Center is omitted for visual simplicity. 


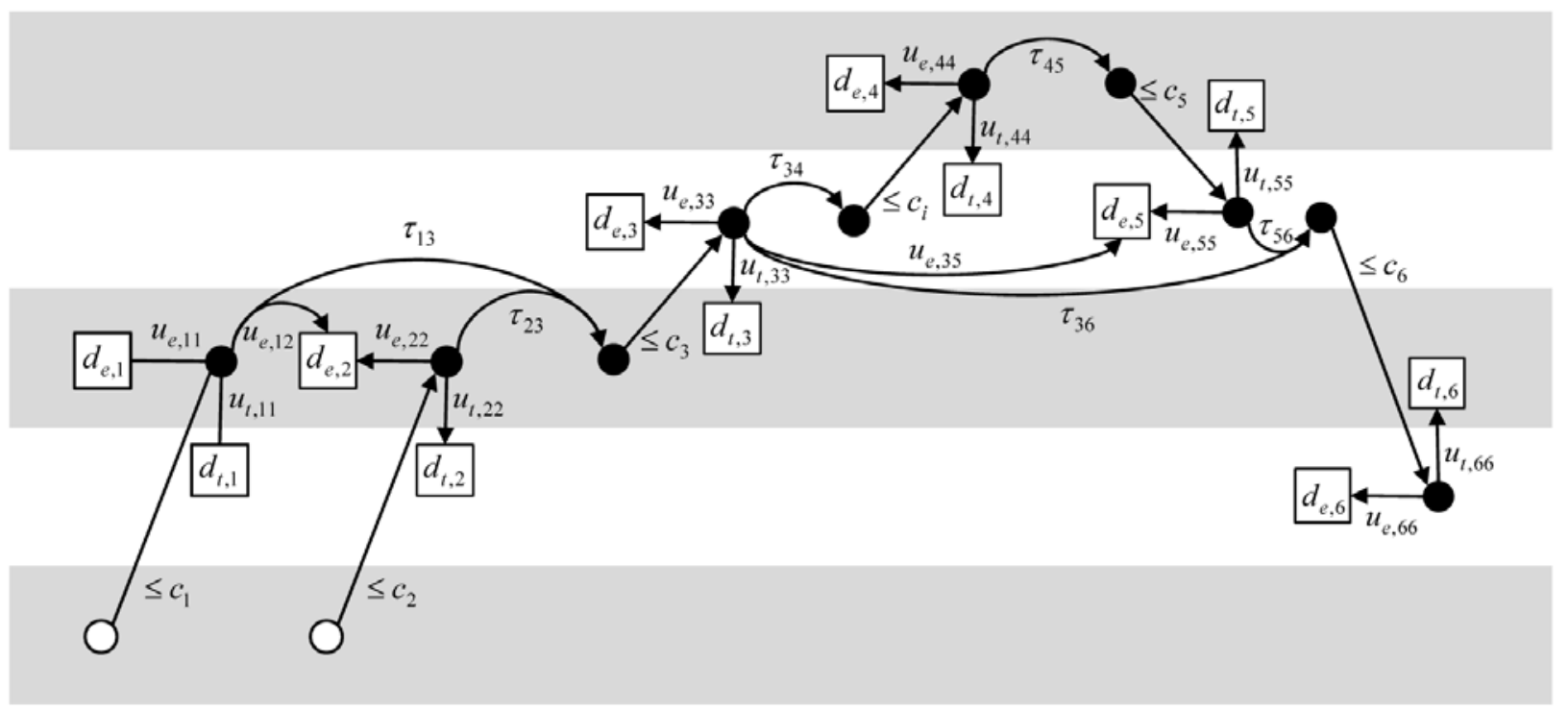

Fig. 4 Composite exploration system network flow graph.

The minimum cost flow problem is solvable in polynomial time for non-integer flows using numerous combinatorial algorithms [11]. This is acceptable given the cargo considered in this analysis is quantized using mass or volume values and is therefore continuous. However, if cargo were to be discretized into logistics carriers such as cargo transfer bags (CTBs) represented with integer flows, the computational complexity of this problem would increase dramatically. Additionally, although system feasibility can be determined in polynomial time, finding the specific components of a network that contribute to the infeasibilities is computationally complex. However, polynomial time algorithms exist to identify a small set of nodes that together exhibit inherent infeasibility (called a minimum witness), which could be a subject of future research [14].

\section{Manifest Optimization Methods}

The transition from space exploration system feasibility to optimal manifesting methods involves only the selection of an objective function. This section describes the construction of relevant exploration system metrics and their application within manifest optimization.

\section{A. Manifest Optimization}

Once a space exploration system is deemed feasible, i.e. at least one valid manifest exists in which all conditions are satisfied, a specific solution may be tailored by using optimization objectives based on some desired logistics 
policy or strategy. A minimum flow manifest, found using LP or flow network methods, can be used as an initial manifest for optimization. The general form of the optimization is shown in Eq. (18), where $\boldsymbol{\mu}$ contains the valid manifest components and $\mathbf{A}, \mathbf{B}, \mathbf{A}_{\mathbf{e q}}$, and $\mathbf{B}_{\mathbf{e q}}$ enforce the feasibility constraints and any additional customized constraints for the specific space exploration system.

$$
\min _{\boldsymbol{\mu}} J(\boldsymbol{\mu}) \text { such that }\left\{\begin{aligned}
\mathbf{A} \cdot \boldsymbol{\mu} & \leq \mathbf{B} \\
\mathbf{A}_{\text {eq }} \cdot \boldsymbol{\mu} & =\mathbf{B}_{\text {eq }} \\
\boldsymbol{\mu} & \geq 0
\end{aligned}\right.
$$

Since the constraints take the form of a linear system of equations, if the objective function $J$ is linear, then the optimization can use linear programming methods (LP), though if $J$ is nonlinear, nonlinear programming methods (NLP) are required. Potential objective functions may seek to minimize costs, distribute risk, improve robustness, or maximize pre-positioned resources, or may consider some combination of these objectives. Nonlinear objective functions benefit from concepts and derived metrics from the M-matrix and D-matrix (described in the following section), which give a sense of cargo dependency between transports.

\section{B. M-matrix and D-matrix Definitions}

Previous work introduced the M-matrix and D-matrix as candidates for use in objective functions [9]. Element $m_{i j}$ represents cargo manifested on flight $i$ consumed during mission $j$ and constitutes the square matrix $\mathbf{M}=\left[m_{i j}\right]_{n: n}$ (for $n$ flights in the campaign). Similarly, element $\delta_{i j}$ represents the dependency of flight $j$ on flight $i$ and forms the matrix $\mathbf{D}=\left[\delta_{i j}\right]_{n n n}$. If there is only a single destination node with no transport demands, the M-matrix is equivalent to $\mathbf{U}_{\mathbf{e}}$. In other cases involving cargo transfer and transportation demands, several variations of the M-matrix and Dmatrix can be devised from the manifest components.

\section{Utilization Manifest Matrix $\left(M_{u}\right)$}

The closest notion to the original M-matrix focuses on the total utilization of cargo, as shown in Eq. (19). In the utilization manifest matrix, element $m_{u, i j}$ captures the cargo manifested on transport $i$ utilized during transport or exploration period $j$ to satisfy a demand. The utilization manifest matrix, however, does not take into account the transfer of cargo between transports. 


$$
\mathbf{M}_{\mathbf{u}}=\mathbf{U}_{\mathrm{e}}+\mathbf{U}_{\mathbf{t}}
$$

\section{2. $\quad$ Flow Manifest Matrix $\left(M_{f}\right)$}

The utilization manifest matrix can be expanded to also include cargo transferred to other transports, as shown in Eq. (20), though certain properties of the matrix (described in detail in [9]) no longer hold. For example, the sum of each column no longer corresponds to the demands for each period due to cargo potentially being transferred multiple times over the course of a campaign. In the flow manifest matrix, element $m_{f, i j}$ captures the cargo manifested on transport $i$ transferred to or utilized during transport or exploration period $j$ to satisfy a demand.

$$
\mathbf{M}_{\mathrm{f}}=\mathbf{U}_{\mathrm{e}}+\mathbf{U}_{\mathrm{t}}+\mathbf{T}
$$

\section{Source Manifest Matrix $\left(M_{s}\right)$}

Each non-source transport receives cargo from a supply chain of transports leading back in time to a source transport. The source manifest matrix captures the chain of transfers between a source transport and the point in which the cargo is utilized to satisfy a demand. Element $m_{s, i j}$ expresses the fraction of cargo that was originally manifested on transport $i$ that contributed to utilization during transport or exploration period $j$ to satisfy a demand.

To calculate the source manifest matrix, the utilization manifest matrix must first be combined with the transfer matrix to decompose the series of individual cargo movements. If a campaign contains $n$ transports, at most $n-1$ decompositions are required to fold all cargo back to the origin node, which is the case for pure sequential transportation architectures. The first decomposition is simply the utilization manifest matrix. For each successive decomposition $k$, the contribution from transport $i$ to $j$ is the prior contribution from each non-source transport $l$ to $j$, weighted in proportion to the fraction of cargo transferred from transport $i$ to $l$, as shown in Eq. (21).

$$
m_{u, i j}^{k}=\left\{\begin{array}{cl}
m_{u, i j} & \text { if } k=1 \\
\sum_{l \mid n_{o, l} \notin S} m_{u, l j}^{k-1} \frac{\tau_{i l}}{\sum_{h} \tau_{h l}} & \text { if } k>1
\end{array}\right.
$$

An important assumption in this formulation is that cargo is transferred to future transports in proportion to the contributions received from previous transports, meaning all transports supplying to a node where cargo may be 
mixed are partially involved in supplying cargo to future transports. Although this assumption does not explicitly hold in practice, the modeling approach presented does not attempt to assign manifest decisions for individual items and therefore does not distinguish cargo based on its origin.

Once the utilization decompositions have been evaluated, the source manifest matrix can be composed. Since the decomposition process does not include transports originating from a source node, the source manifest matrix sums contributions from all decompositions with a source transport contributing cargo utilization, as shown in Eq. (22).

$$
m_{s, i j}= \begin{cases}\sum_{k} m_{u, i j}^{k} & \text { if } n_{o, i} \in S \\ 0 & \text { otherwise }\end{cases}
$$

\section{Utilization Dependency Matrix $\left(D_{w}\right)$}

In the absence of backorders, the utilization dependency matrix captures the immediate nature of cargo utilization as carried-along or pre-positioned. Element $\delta_{u, i j}$ represents the fraction of cargo from transport $i$ that contributes to utilization during transport or exploration period $j$, as shown in Eq. (23).

$$
\delta_{u, i j}=\frac{m_{u, i j}}{\sum_{i} m_{u, i j}}
$$

\section{Source Dependency Matrix $\left(D_{s}\right)$}

Analogous to the utilization dependency matrix, the source dependency matrix captures the dependency of each transport on the source transports. Element $\delta_{s, i j}$ represents the fraction of cargo from source transport $i$ that contributes to utilization during transport or exploration period $j$, as shown in Eq. (24).

$$
\delta_{s, i j}=\frac{m_{s, i j}}{\sum_{i} m_{s, i j}}
$$

\section{Transportation System Analysis}


In addition to the M- and D-matrices, several other visualizations and derivative exploration system analysis metrics are described below. These metrics are useful for understanding space transportation networks and in the formulation of optimization objective functions.

\section{Global Aggregate Feasibility}

A necessary but not sufficient condition for exploration system feasibility can be expressed by global aggregate feasibility. To achieve feasibility from a global perspective, the cumulative capacity from all source transports must exceed the total cumulative demands (both transport and exploration) at all points in time. This can be expressed mathematically using Eq. (25), but is more useful when visualized as a plot over time. The exploration system may be feasible if the cumulative source capacity line always exceeds the cumulative global demand line. Figure 5 illustrates the global aggregate feasibility of the dual-launch sortie defined in Table 1.

$$
\sum_{j \leq i \mid n_{o, j} \in S} c_{j} \geq \sum_{j \leq i} d_{e, j}+d_{t, j} \forall i
$$

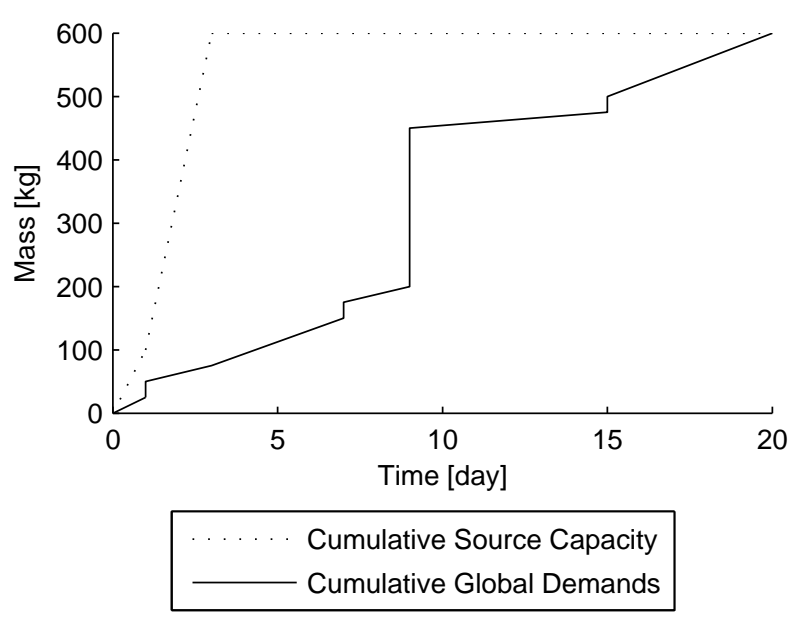

Fig. 5 Example global aggregate feasibility plot.

\section{Exploration System Network}

The exploration system network is a visualization showing the entire space exploration system definition in a time-expanded network similar to a bat chart. The transport lines are weighted in proportion to the demands occurring in transport and circles are weighted in proportion to exploration demands. The representation of nodes and arcs is similar to the network flow formulation presented in Fig. 4, but simplified to omit demand sinks and 18

American Institute of Aeronautics and Astronautics 
manifesting arcs. Visual inspection can help identify bottlenecks in the transportation network that are not uncovered by global aggregated feasibility checks. Figure 6 illustrates a sample exploration system network based on the dual-launch sortie defined in Table 1.

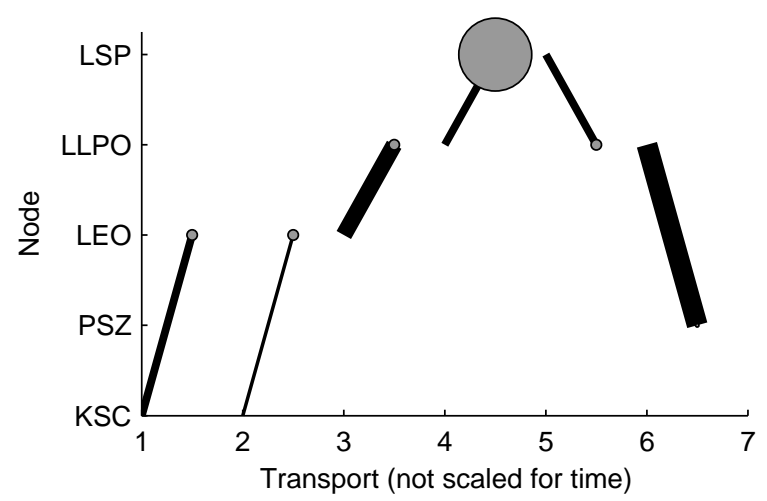

Fig. 6 Example exploration system network with weighted arcs and nodes.

\section{Transport Criticality Index (TCI)}

The $\delta_{i j}$ components of the D-matrices define the interdependence between transports, but they do not identify the impact of a transport on the overall exploration system. Transport criticality can be determined both for source dependency $\left(\delta_{s, i j}\right)$ and utilization dependency $\left(\delta_{u, i j}\right)$ components. Source dependency traces criticality back to the source transports (i.e. launches) while the utilization dependency identifies the criticality for a single link within the exploration system.

Transport criticality is comprised of two components. The first component measures the extent of dependence on a transport based on cargo. It is calculated for transport $i$ using Eq. (26).

$$
\sum_{j} \delta_{\cdot, i j}
$$

The second component measures the breadth of transports supplied. Transport $j$ is supplied by $i$ if it receives utilization or transfer cargo, i.e. $\delta_{; ;}$is nonzero. It is calculated for transport $i$ using Eq. (27).

$$
\sum_{j} n_{\cdot, i j} \text { where } n_{\cdot, i j}=\left\{\begin{array}{l}
1 \text { if } \delta_{\cdot, i j}>0 \\
0 \text { if } \delta_{\cdot, i j}=0
\end{array}\right.
$$


A transport criticality (TC) plot is obtained by plotting the dependency breadth against the dependency extent. A line tracing the points $\sum_{j} \delta_{\cdot, i j}=\sum_{j} n_{, i j}$ serves as an upper bound for all transport criticalities. If a transport lies on this line on the TC plot, it means that it completely supplies all dependent transports, i.e. it is the sole carrier of the cargo that is subsequently needed by other transports that depend on it. The most crucial transports will be placed in the upper-right of the plot, indicating a high extent of supply to a large number of transports. A sample TC plot for the dual-launch sortie is illustrated in Fig. 7 showing both source dependency and utilization dependency. In this example, transport 2 is the most critical source transport and transport 3 is the most critical single-transport link.

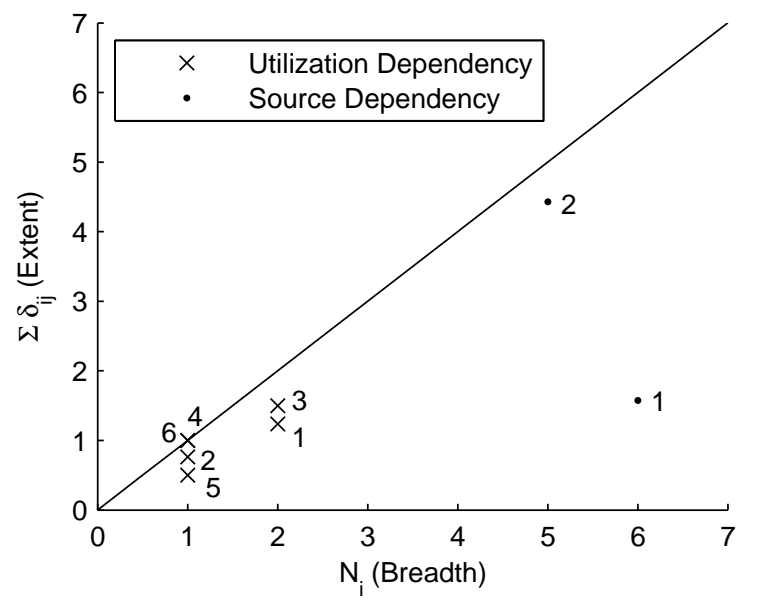

Fig. 7 Example transport criticality (TC) plot.

The composite transport criticality index (TCI) is defined as the Euclidean distance from the origin to a breadthextent pair, shown in Eq. (28). This formulation places equal emphasis on breadth and extent as components of transport criticality.

$$
T C I_{\cdot, i}=\sqrt{\left(\sum_{j} \delta_{\cdot, i j}\right)^{2}+\left(\sum_{j} n_{\cdot, i j}\right)^{2}}
$$

\section{Capacity Utilization Index (CUI)}

The capacity utilization index provides the ratio between cargo carried and a transport's total cargo capacity. It is calculated using the flow M-matrix and the transport capacities, as shown in Eq. (29). Though it may be desirable to maximize CUI across all transports, reaching the maximum value of 1 is nearly impossible for most exploration 
systems because the demand constraint equalities and the flow conservation constraints do not allow a build-up of non-utilized cargo. In practice, any excess capacity after cargo manifesting could be used for additional payload (such as scientific equipment that is not explicitly modeled as demand) or contingency cargo. Once defined, these additional payload items would eliminate the excess capacity and result in a higher CUI.

$$
C U I_{i}=\frac{\sum_{j} m_{f, i j}}{c_{i}}
$$

\section{Logistics Strategy Index (LSI)}

The logistics strategy index is a ratio that indicates the amount of cargo prepositioned for future use versus the amount of cargo that is utilized during transport or in the exploration period that immediately follows. A value of 0 indicates all manifested cargo is used during the transport (or during its immediate exploration mission duration), i.e. it is a "carry-along" transport. A value of 1 indicates all manifested cargo is used during future transports and associated explorations, in other words it does pure pre-positioning. At the exploration level, the exploration LSI (eLSI) is calculated using Eq. (30).

$$
e L S I_{i}=\frac{\sum_{j<i} m_{u, j i}}{\sum_{j} m_{u, j i}}
$$

At the system level, the system LSI (sLSI) is calculated using Eq. (31) summing over all transports in the campaign.

$$
s L S I=\frac{\sum_{i} \sum_{j<i} m_{u, j i}}{\sum_{i} \sum_{j} m_{u, j i}}
$$

\section{Case Study: Lunar Surface Exploration}

To demonstrate the optimization of cargo manifests in a relevant space exploration system, a sample lunar surface exploration system based on NASA human spaceflight program concepts is presented. The exploration 
system is defined and its feasibility determined before optimizing cargo manifests under several objective functions. In this analysis, kilograms are used to set mass capacity limits for transports and demand quantities, though cubic meters (or similar units) could alternatively be used to capture volume constraints.

\section{A. Case Study Summary}

As of late 2009, the most current iteration of the lunar surface architecture released by NASA's Lunar Surface Systems Project Office (LSSPO) and the Constellation Architecture Team - Lunar (CxAT-Lunar) was Scenario 12 $[15,16]$. In the described scenario architecture, successive missions (at a rate of about three per year) deliver infrastructure components to an outpost on the rim of Shackleton Crater at the Lunar South Pole. As infrastructure and supplies grow, explorations of increasing duration are enabled, leading up to continuous human presence. Due to the maturity of the campaign architecture, the exploration benefits from detailed element models are based on prior analysis.

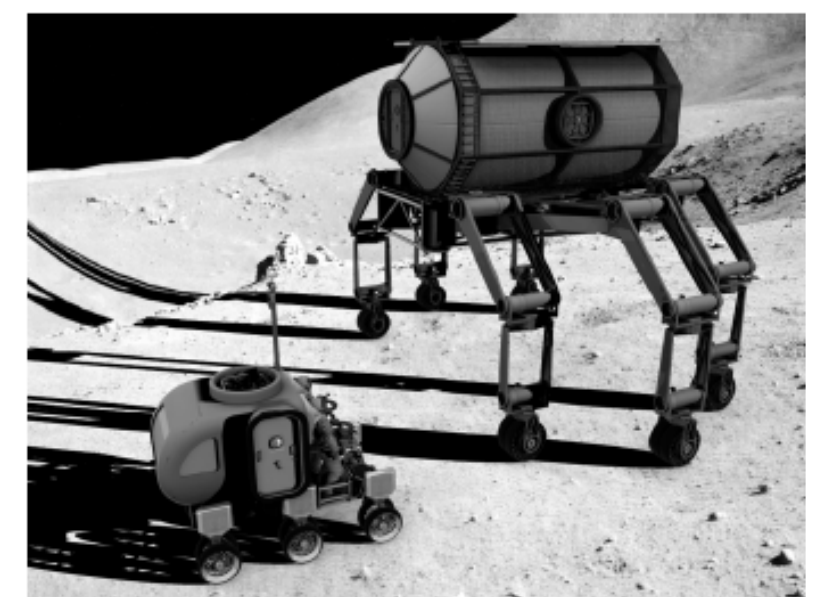

Fig. 8 Lunar surface mobility elements: LER (left) and ATHLETE (right), adapted from [15].

One of the interesting aspects of Scenario 12 from a manifesting perspective is an emphasis on surface mobility elements for performing excursions to nearby locations, illustrated in Fig. 8. The lunar electric rover (LER) carries a crew up to 200 kilometers on one charge. The all-terrain hex-limbed extra-terrestrial explorer (ATHLETE) is capable of traversing difficult terrain while carrying a payload as large as a habitat or logistics module. In this case study the LER and the ATHLETE will be used to perform several surface excursions requiring additional manifesting to satisfy demands during transport and remote exploration.

\section{B. Transportation System Definition}


The exploration system is comprised of 32 transports - 17 landings on the lunar surface (8 cargo and 9 crewed with four astronauts each) and 15 surface transports supporting excursions. A mission is notionally defined as the period of time between subsequent crewed or cargo landings inclusive of all surface transports. To simplify the exploration system definition, return transports for crewed missions are omitted as no demands are considered after ascent from the lunar surface. The complete exploration system definition is included in Appendix A.

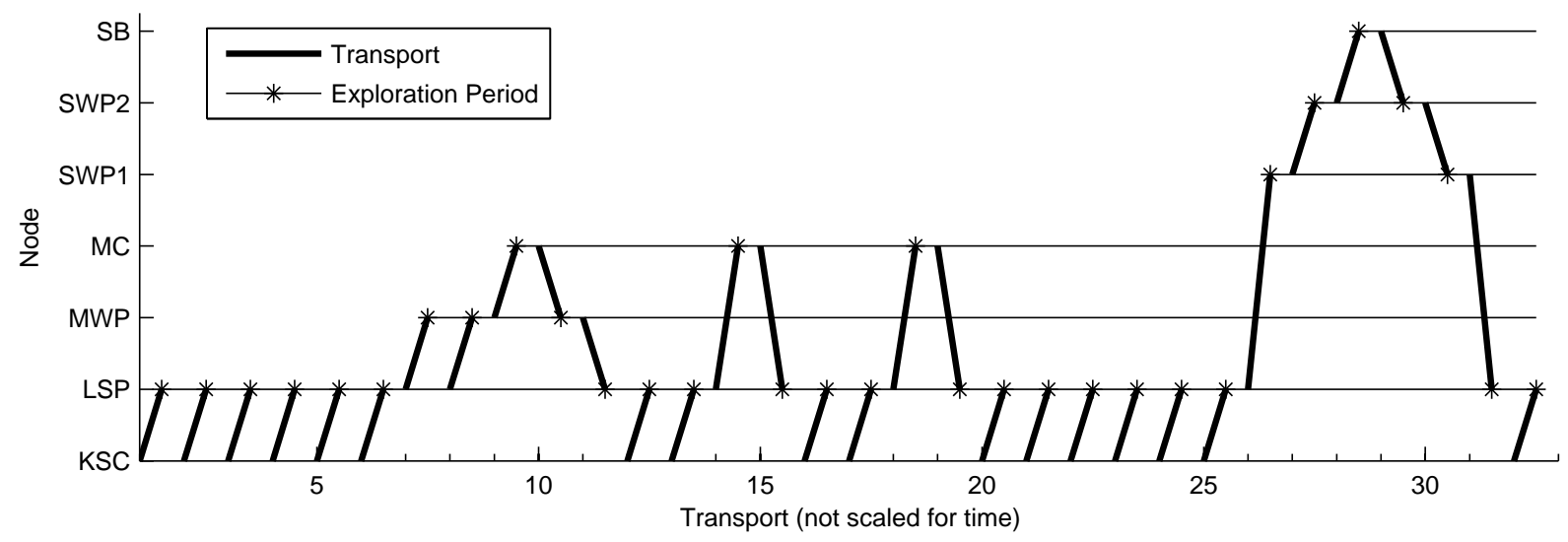

Fig. 9 Lunar exploration system bat chart.

The first few missions establish basic infrastructure at the Lunar South Pole (LSP). Mission 6 (transports 6-11) performs a surface excursion to the nearby Malapert Crater (MC) using LERs. A logistics LER traverses to a waypoint (MWP) to pre-position contingency supplies a few days before the four crew members leave in two LERs. After reaching the waypoint, the crew continues two days to Malapert Crater and performs a 7-day local exploration. After exploration, the crew returns to the MWP waypoint, retrieves the logistics LER, and continues back to the base.

Missions 8 (transports 13-15) and 10 (transports 17-19) also include surface excursions to Malapert Crater, but without utilizing the waypoint. Mission 8 performs a seven-day remote exploration while pre-positioning a logistics carrier. Mission 10 performs a 14-day remote exploration before returning to the base with the logistics carrier.

Finally, mission 16 (transports 25-31) is largely comprised of an extended 83-day surface excursion to the Schrodinger Basin (SB). A convoy of two crewed LERs and two ATHLETEs, one carrying a portable excursion module (PEM) and one carrying a pressurized logistics module (PLM), passes two waypoints (SWP1, SWP2) on the way to the destination. The total transit to Schrodinger Basin spans 24 days, remote exploration 14 days, and the return transit 45 days. 
To estimate demands for resources during surface transports and local exploration including the effects of ISRU and ECLSS processes, the lunar exploration system was analyzed using SpaceNet, an open source integrated modeling and simulation software tool [10]. An underlying discrete event simulator enables the instantiation of element models and definition of transports between nodes. Demands for resources are based on demand models both at the mission and element level. Mission-level demand models were used to establish required masses of scientific payloads. Element-level demand models were used to build up the remaining aggregated demand mass. Demands include crew consumables totaling 7.5 kilograms per person per day (after considering ECLSS processes) and spares for major infrastructure elements estimated at $10 \%$ of the element mass per year during active periods and 5\% during inactive periods. SpaceNet demand integration also offsets crew demands for oxygen (2 kilograms per day) with any available ISRU plant oxygen, which is produced at a rate of 1000 kilograms per plant per year after plants are delivered in missions 6 and 12.

The lunar exploration system definition implies a build-up of a surface infrastructure at the LSP base. Supporting large amounts of infrastructure, along with longer crewed durations for later missions, creates a non-linear composite demand model with significantly more demands towards the end of the modeling timeframe. Figure 10 illustrates the increasing aggregated exploration and transportation demands throughout the scenario as more infrastructure to support is delivered. Detailed demand data is available in Appendix A.

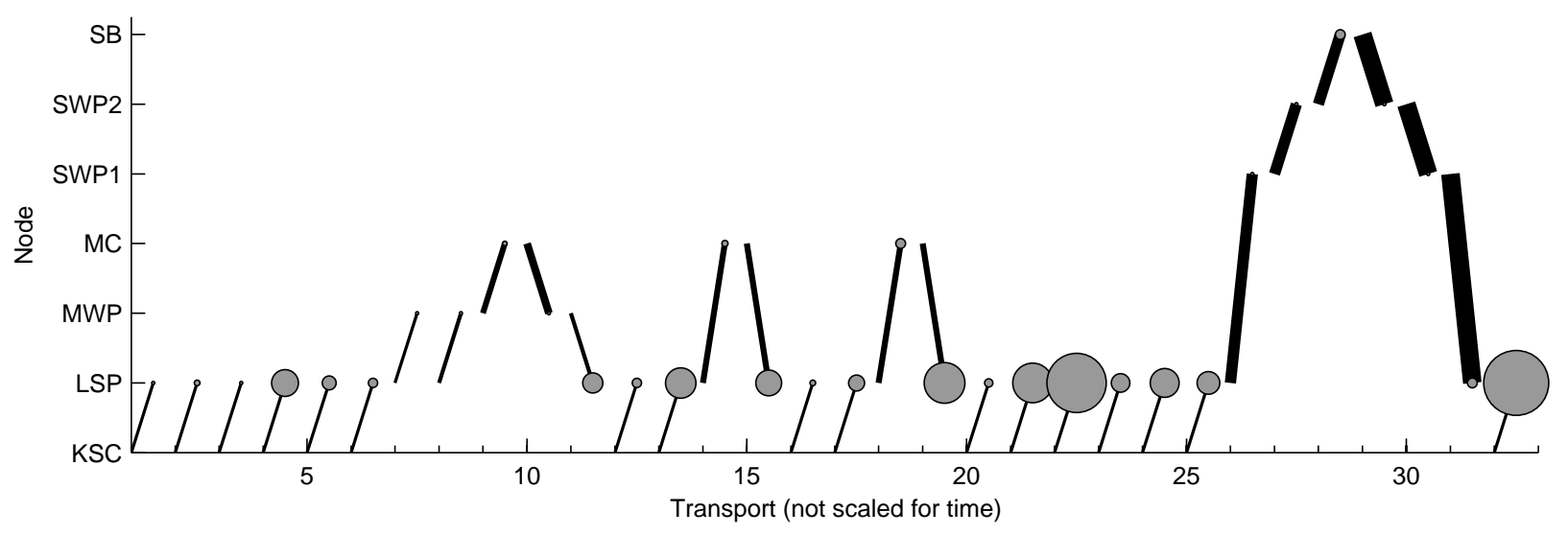

Fig. 10 Lunar exploration transportation network illustrating increasing demands.

\section{Transportation System Feasibility}


The lunar exploration system meets the necessary feasibility condition from the global perspective, illustrated in Fig. 11. For many of the intermediate missions there is a margin between the maximum source capacity and global demands, though the margin decreases substantially after mission 14.

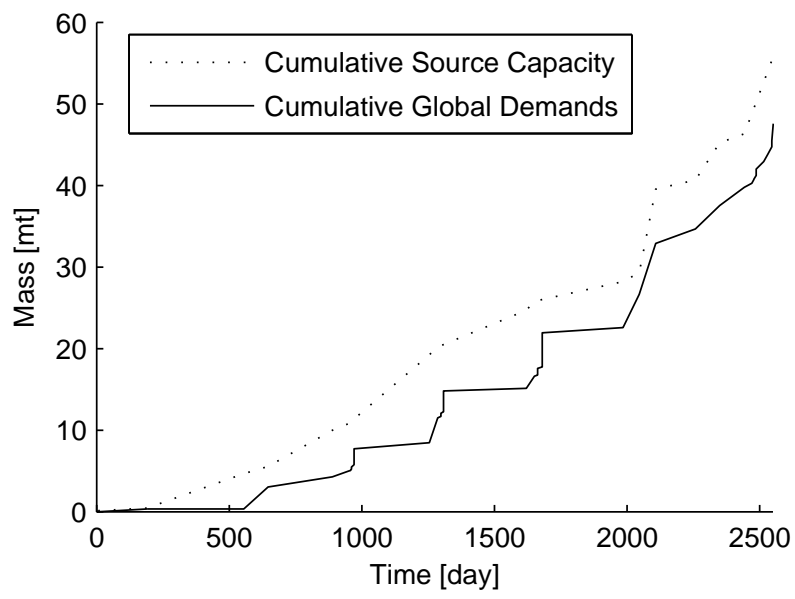

Fig. 11 Global aggregate feasibility plot.

To verify system feasibility, Eq. (17) is used to formulate a LP problem. Under the validity conditions imposed by Eq. (12), $\boldsymbol{\mu}$ contains a total of 352 variables. Using the function linprog within the Matlab Optimization Toolbox $^{\mathrm{TM}}$ and an objective function of $J(\boldsymbol{\mu})=\sum_{i} \mu_{i}$ a minimum flow manifest is found, establishing the lunar exploration system feasibility. The resulting TC plot for the minimum flow manifest is illustrated in Fig. 12. It is interesting to note two groups of transports. One group is clustered in the top right (12, 3, 24, 22 and 5: all cargo transports) the other group is clustered in the bottom middle (such as 17, 13, 25, 4: mostly crewed transports). Transports in the top right cluster are very critical since they are supplying a large fraction of cargo to a large number of other transports (up to 14 other transports) as shown in Fig. 10. The bottom middle cluster supplies a much lower fraction of cargo to a fewer number of transports. 


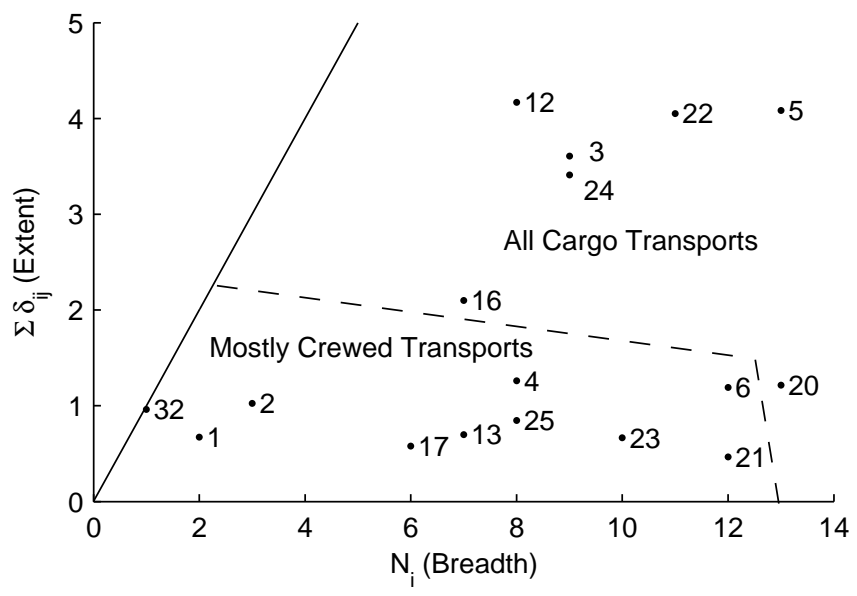

Fig. 12 Minimum flow manifest source TC plot $(\mathrm{sLSI}=\mathbf{0 . 5 4 7 2})$.

\section{Dormant Cargo Limit Sensitivity Analysis}

To expand upon the basic manifest model while reducing the problem scale, additional constraints limit cargo lifetime. As resources are treated as lumped mass, there is no available constraint for absolute lifetime (i.e. time between source transport and utilization), though a limit on dormant cargo time can be imposed as a proxy. Dormant cargo spans are defined as the time between cargo delivery and subsequent utilization or transfer to a later transport.

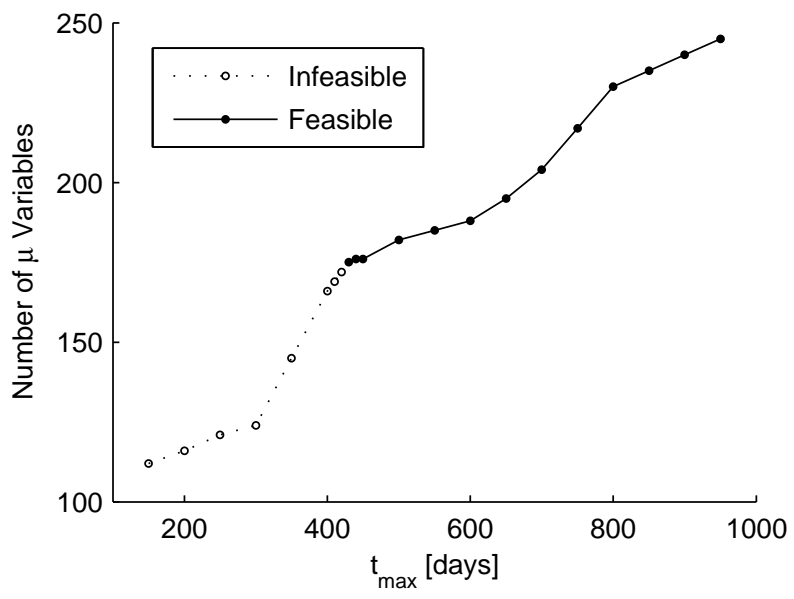

Fig. 13 Exploration feasibility sensitivity to cargo limitations.

A variation of the validity constraints introduced in Eq. (12) is proposed to implement a dormant cargo limit $t_{\max }$. The modified set of validity constraints are summarized in Eq. (32). This extra constraint creates an upper bound to pre-positioning options and can significantly reduce the number of manifest variables as well as identifying limits of feasibility. 


$$
\boldsymbol{\mu} \equiv\left\{\mathbf{U}_{\mathbf{e}}, \mathbf{U}_{\mathbf{t}}, \mathbf{T}\right\} \forall\left\{\begin{array}{l}
u_{e, i j} \mid n_{d, i}=n_{d, j} \\
u_{e, i j} \mid 0 \leq t_{a, j}-t_{a, i} \leq t_{\max } \\
u_{t, i j} \mid i=j \\
\tau_{i j} \mid n_{d, i}=n_{o, j} \\
\tau_{i j} \mid 0 \leq t_{d, j}-t_{a, i} \leq t_{\max }
\end{array}\right.
$$

A sensitivity analysis is performed to identify the exploration system feasibility dependence on dormant cargo limits. Both the number of remaining manifest variables and the resulting feasibility are summarized in Fig. 13. These results indicate that certain cargo must be capable of dormant periods of at least 430 days to achieve logistical feasibility for the lunar exploration system.

\section{E. Cargo Manifest Optimization}

Building off of the previous sensitivity analysis, a dormant cargo limit of 600 days is instituted for the cargo manifest optimization. The resulting manifest vector is reduced from 354 to 188 variables, substantially decreasing the nonlinear optimization computation time.

As a system-level measure of the underlying strategy for cargo manifests, the sLSI metric will form the basis of two optimization objective functions. The pre-position strategy seeks to maximize sLSI, attempting to decouple each transport with its immediate exploration demands. The alternative carry-along strategy seeks to minimize sLSI, attempting to decouple exploration demands from as many transports as possible. While the first objective increases logistical robustness at exploration nodes by building up local safety stock, the second method decreases the likelihood that crew and cargo will be separated due to unforeseen events.

The optimization was performed using the fmincon function within the Matlab Optimization Toolbox ${ }^{\mathrm{TM}}$ using a minimum flow manifest as an initial solution and a functional tolerance of $10^{-9}$ as a convergence criterion for finding a local extrema. The optimized feasible sLSI for the pre-position strategy was 0.6692 and for the carry-along strategy was 0.3320 .

Comparative manifest analysis will use the metrics previously defined as the raw manifest matrices are unwieldy to process. The source TC plots, shown in Fig. 14, illustrate the general differences between the two strategies. Corresponding transports occupy similar regions between the two plots, due to the nature of cargo versus crewed transports, but the pre-position strategy stretches transports out over a larger breadth, indicating that the average 
transport is serving more exploration periods. This highlights a fundamental tradeoff between the carry-along and pre-position strategies: pre-positioning increases transport criticality as defined.
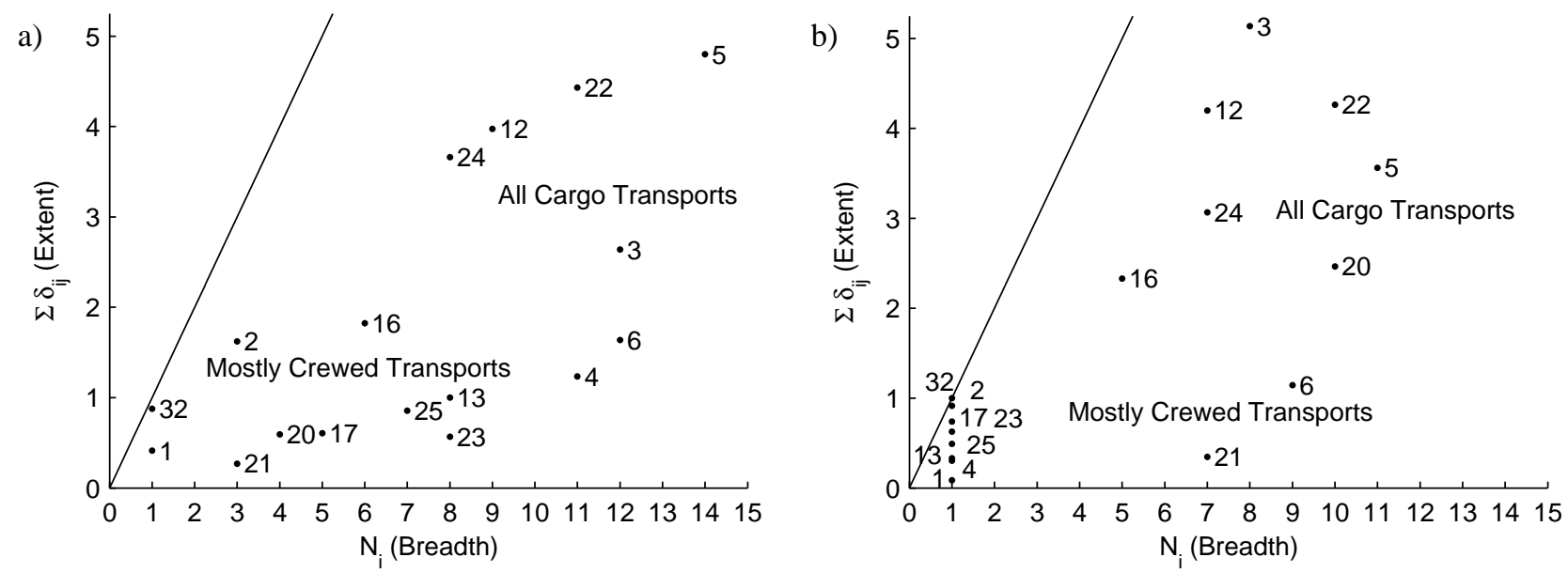

Fig. 14 Optimized manifest source TC plots: (a) pre-position strategy, sLSI=0.6692; (b) carry-along strategy, SLSI $=\mathbf{0 . 3 3 2 0}$.

The utilization transport criticality indices, shown in Fig. 15, emphasize the same point. Though only tracking one iteration of resource flow, most transports under the pre-position strategy have a higher criticality. The mean TCI for the pre-position transports is 3.02 with a standard deviation of 1.48 compared to a mean TCI of 1.99 and a standard deviation of 1.39 for the carry-along strategy.

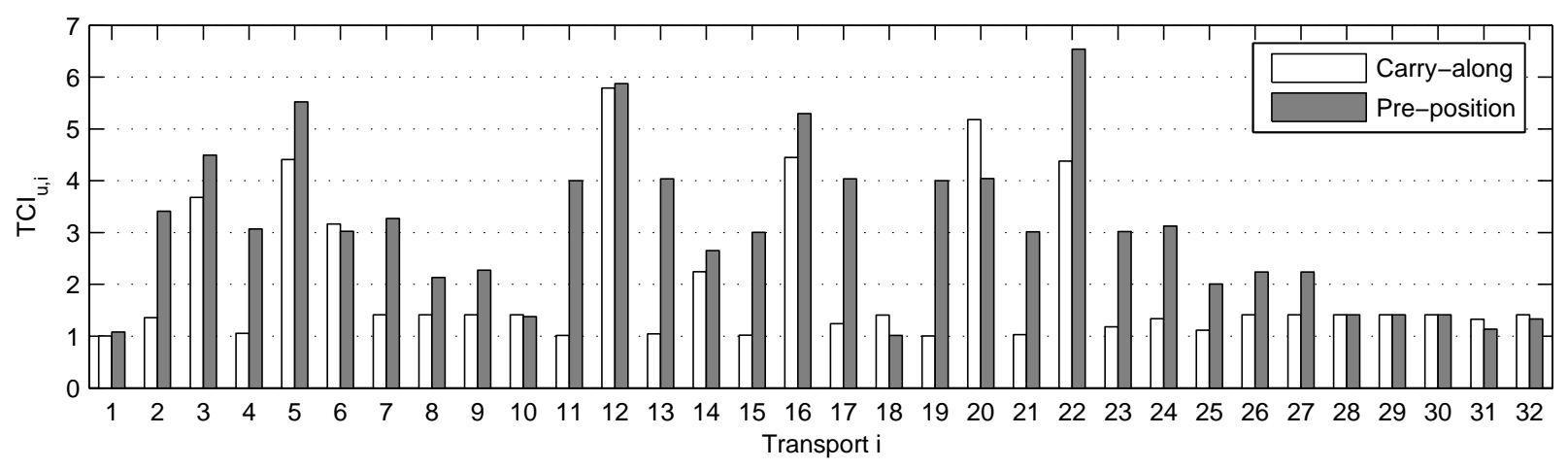

Fig. 15 Comparison of utilization transport criticality indices.

The exploration-level logistics strategy indices (eLSI), shown in Fig. 16, highlight the selection of prepositioning for each transit using the system-level pre-position strategy. Explorations 1, 7, 9, 26, 27, and 28 cannot take advantage of pre-positioned cargo as these are the first transport to a new location (LSP, MWP, MC, SWP1, SWP2, and SB respectively), therefore their eLSI is zero by default. 


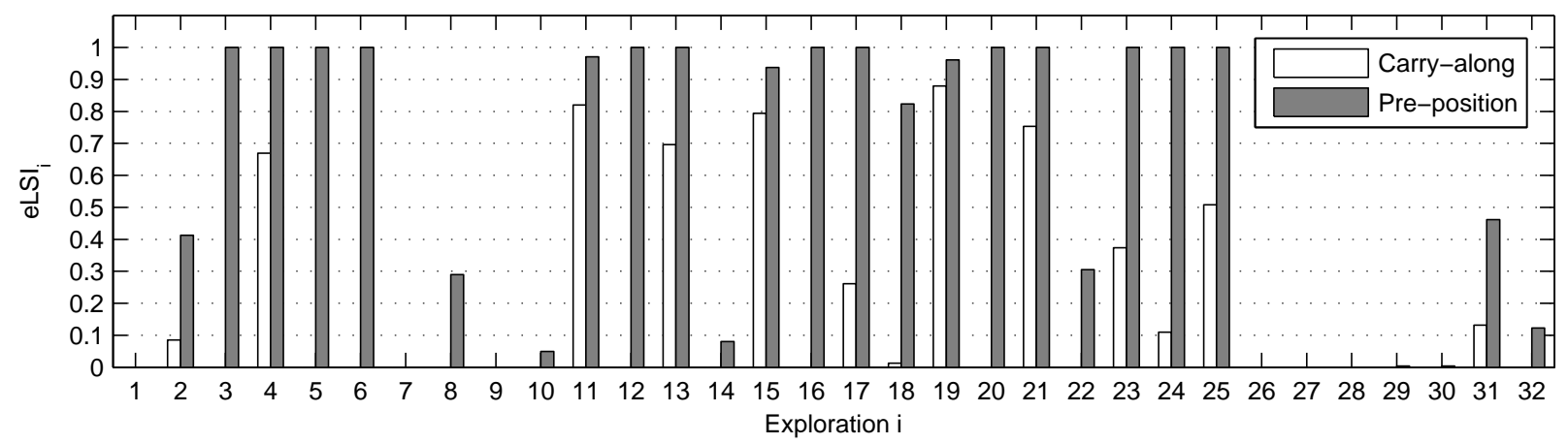

Fig. 16 Comparison of exploration logistics strategy indices.

The transport capacity utilization indices, shown in Fig. 17, are not much different between the two strategies except for the departing surface excursion transports $(7,8,14,18$, and 25). Not constrained to find a minimum flow manifest, the carry-along strategy transfers additional cargo over the entire excursion, some of which is used in place of pre-positioned cargo opportunities. Which of the two manifesting strategies to use depends on the relative assessment of risk for emergence of undersupply situations at the LSP base (driven by reliability of in-space cargo transports) versus the likelihood of crew and cargo being separated during surface transports (reliability of crew transports).

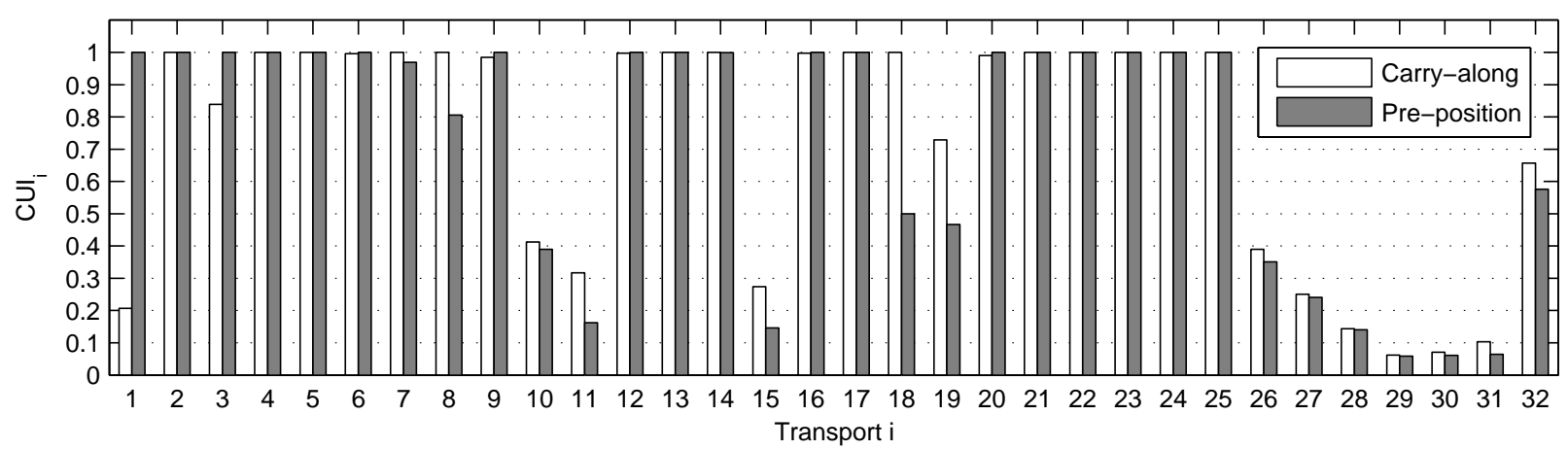

Fig. 17 Comparison of capacity utilization indices (CUI).

\section{Conclusion}

This paper has shown how a matrix-based approach may be used to model and determine optimal manifests for multi-node space exploration systems. Logistical feasibility can be determined using linear programming or flow network graph methods to find a minimum flow manifest. Finding an optimal manifest subject to a desired objective function requires the use of linear or nonlinear programming methods and a system objective function. Extensions of the basic modeling framework can also be used to determine feasibility sensitivity to other constraints such as 29

American Institute of Aeronautics and Astronautics 
dormant cargo limits. Strategies of maximizing pre-positioned and carry-along cargo were presented as bookend cases and additional objective functions balancing the two cases are a topic for further research.

For simplicity and tractability under optimization, the analysis has thus far treated all demands as an aggregated resource without differentiation between types of cargo. It may be possible to maintain tractability and distinguish cargo by functional class of supply, a concept implemented in other logistical models [17]. A natural extension of this manifest model would establish sources and sinks for each class of supply individually with capacity constraints enforced over all classes manifested on a transport. This extension may enable the explicit modeling of resource transformations to waste, recycling of waste back to useful resources, and the mining and utilization of in-situ resources. A detailed manifesting model may also assign different priority levels for different resources. For example, crew provisions and spares needed for sustenance of crew and keeping vital equipment running would be of highest priority followed by other types of supplies.

In addition to distinguishing between cargo class of supply, future modeling extensions may distinguish between cargo stowage environments. Many spacecraft have aggregate mass and volume capacity limits as well as constraints on masses of pressurized, unpressurized, gas, and liquid cargo that form a coupled system capacity. For example, a resupply vehicle may have capacity for 1500 kilograms of pressurized dry cargo and 1540 kilograms of liquid cargo, but only an aggregate capacity of 2350 kilograms across both environments. A coupled manifesting decision is required to determine the distribution between each cargo environment. Another extension of this work would allow for both Monte Carlo simulation of demand models as well as discrete event simulation of failed or delayed transports. The distribution of outcomes from such probabilistic models would allow selection of prepositioning versus carry-along manifesting strategies based on a quantitative assessment of various campaign uncertainties.

Finally, integration with the SpaceNet modeling environment is desired to improve integrated space exploration analysis. Many of the finer-level modeling details, including functional class of supply demand breakdowns and cargo environment differentiation currently exist or are under development within SpaceNet. Furthermore, the existing feasibility determination and cargo manifesting methods within SpaceNet are tedious and are in need of robust automation, similar to what could be provided with the presented methods. Future work is targeted at integrating the matrix-based optimal manifesting methods with the SpaceNet model. 


\section{Appendix A}

Table 2 Lunar exploration system definition for lunar exploration campaign

\begin{tabular}{|c|c|c|c|c|c|c|c|c|c|}
\hline Mission & $i$ & $n_{o, i}$ & $\begin{array}{c}t_{d, i} \\
{[\text { day] }}\end{array}$ & $n_{d, i}$ & $\begin{array}{c}\boldsymbol{t}_{a, i} \\
{[\mathrm{day}]}\end{array}$ & $\begin{array}{c}c_{i} \\
{[\mathrm{~kg}]}\end{array}$ & $\begin{array}{c}d_{t, i} \\
{[\mathrm{~kg}]}\end{array}$ & $\begin{array}{r}\boldsymbol{d}_{e, i} \\
{[\mathrm{~kg}]}\end{array}$ & Comments \\
\hline 1.0 & 1 & KSC & 0 & LSP & 6 & 140 & 0 & 0 & Uncrewed checkout exploration \\
\hline 2.0 & 2 & KSC & 184 & LSP & 190 & 310 & 0 & 339 & 7-day crewed sortie \\
\hline 3.0 & 3 & KSC & 549 & LSP & 555 & 4210 & 0 & 13 & Cargo delivery (incl. 2 LERs) \\
\hline 4.0 & 4 & KSC & 641 & LSP & 647 & 890 & 0 & 2692 & 14-day crewed exploration \\
\hline 5.0 & 5 & KSC & 883 & LSP & 889 & 4420 & 0 & 1227 & Cargo delivery (incl. 2 LERs) \\
\hline 6.0 & 6 & KSC & 944 & LSP & 950 & 825 & 0 & 727 & 28-day crewed exploration (total) \\
\hline 6.1 & 7 & LSP & 953 & MWP & 954 & 430 & 3 & 8 & Logistics LER pre-position at waypoint \\
\hline 6.2 & 8 & LSP & 957 & MWP & 958 & 690 & 54 & 22 & Crewed LERs travel to Malapert waypoint \\
\hline 6.3 & 9 & MWP & 958 & MC & 961 & 690 & 161 & 215 & Crewed LERs travel to Malapert Crater \\
\hline 6.4 & 10 & $\mathrm{MC}$ & 965 & MWP & 970 & 690 & 269 & 14 & Crewed LERs travel to Malapert waypoint \\
\hline 6.5 & 11 & MWP & 970 & LSP & 971 & 1120 & 57 & 1922 & Crewed and log. LERs return to base \\
\hline 7.0 & 12 & KSC & 1249 & LSP & 1255 & 8460 & 0 & 727 & Cargo delivery (incl. 2 tri-ATHLETEs) \\
\hline 8.0 & 13 & KSC & 1280 & LSP & 1286 & 930 & 0 & 3059 & 28-day crewed exploration (total) \\
\hline 8.1 & 14 & LSP & 1295 & $\mathrm{MC}$ & 1298 & 1640 & 169 & 394 & Crewed and log. LER travel to Malapert \\
\hline 8.2 & 15 & MC & 1305 & LSP & 1308 & 2040 & 169 & 2549 & Crewed and log. LER return to base \\
\hline 9.0 & 16 & KSC & 1614 & LSP & 1620 & 4500 & 0 & 324 & Cargo delivery (incl. 1 tri-ATHLETE) \\
\hline 10.0 & 17 & KSC & 1645 & LSP & 1651 & 1100 & 0 & 1489 & 50-day crewed exploration (total) \\
\hline 10.1 & 18 & LSP & 1660 & MC & 1663 & 950 & 170 & 792 & Crewed and log. LER travel to Malapert \\
\hline 10.2 & 19 & MC & 1677 & LSP & 1680 & 720 & 170 & 4200 & Crewed and log. LER return to base \\
\hline 11.0 & 20 & KSC & 1979 & LSP & 1985 & 2425 & 0 & 622 & Cargo delivery (incl. 1 tri-ATHLETE) \\
\hline 12.0 & 21 & KSC & 2040 & LSP & 2046 & 1100 & 0 & 4082 & 110-day crewed exploration \\
\hline 13.0 & 22 & KSC & 2102 & LSP & 2108 & 10230 & 0 & 6268 & Cargo delivery \\
\hline 14.0 & 23 & KSC & 2252 & LSP & 2258 & 1100 & 0 & 1756 & 180-day crewed exploration \\
\hline 15.0 & 24 & KSC & 2344 & LSP & 2350 & 4600 & 0 & 2878 & Cargo delivery \\
\hline 16.0 & 25 & KSC & 2436 & LSP & 2442 & 1100 & 0 & 2236 & 180-day crewed exploration (total) \\
\hline 16.1 & 26 & LSP & 2463 & SWP1 & 2471 & 14100 & 478 & 12 & $\begin{array}{l}\text { Crewed LERs and ATHLETEs travel to } \\
\text { Schrodinger waypoint } 1\end{array}$ \\
\hline 16.2 & 27 & SWP1 & 2471 & SWP1 & 2479 & 14750 & 477 & 7 & $\begin{array}{l}\text { Crewed LERs and ATHLETEs travel to } \\
\text { Schrodinger waypoint } 2\end{array}$ \\
\hline 16.3 & 28 & SWP2 & 2479 & SB & 2487 & 15430 & 475 & 786 & $\begin{array}{l}\text { Crewed LERs and ATHLETEs travel to } \\
\text { Schrodinger basin }\end{array}$ \\
\hline 16.4 & 29 & SB & 2501 & SWP1 & 2516 & 15430 & 897 & 3 & $\begin{array}{l}\text { Crewed LERs and ATHLETEs travel to } \\
\text { Schrodinger waypoint } 2\end{array}$ \\
\hline 16.5 & 30 & SWP2 & 2516 & SWP1 & 2531 & 14750 & 900 & 3 & $\begin{array}{l}\text { Crewed LERs and ATHLETEs travel to } \\
\text { Schrodinger waypoint } 1\end{array}$ \\
\hline 16.6 & 31 & SWP1 & 2531 & LSP & 2546 & 14100 & 903 & 775 & Crewed LERs and ATHLETEs return to base \\
\hline 17.0 & 32 & KSC & 2557 & LSP & 2563 & 10500 & 0 & 6895 & Cargo delivery \\
\hline
\end{tabular}

\section{References}

[1] Shull, S., Integrated Modeling and Simulation of Lunar Exploration Campaign Logistics, S.M. Thesis, Department of Aeronautics and Astronautics, Massachusetts Institute of Technology, Cambridge, Massachusetts, 2007.

[2] Carr, D. L., Into the Unknown: The Logistics Preparation of the Lewis and Clark Expedition, Combat Studies Institute Press, Leavenworth, Kansas, 2004. http://purl.access.gpo.gov/GPO/LPS59546 [retrieved 10 October 2010]. 
[3] Pugh, L. G. C., “The Logistics of the Polar Journeys of Scott, Shackleton and Amundsen,” Proceedings of the Royal Society of Medicine, Vol. 65 No. 1, 1972, pp. 42-47.

[4] Blackadar, R., G., "Field Methods and Logistics; Arctic Canada,” Geology of the Arctic; proceedings held January 11-13, 1960 under the auspices of the Alberta Society of Petroleum Geologists, University of Toronto Press, Toronto, 1961, pp. 1095-1101.

[5] Kress, M., Operational Logistics: The Art and Science of Sustaining Military Operations, Kluwer Academic Publishers, Boston, Massachusetts, 2002, pp. 17-36.

[6] Baker, S. F., Morton, D. P., Rosenthal, R. E., Williams, L. M., “Optimizing Military Airlift,” Operations Research, Vol. 50, No. 4, 2002, pp. 582-602. doi: 10.1287/opre.50.4.582.2864

[7] Brandimarte P., Zotteri G., Introduction to Distribution Logistics, John Wiley \& Sons, New Jersey, 2007.

[8] Klingman, D., Mote, J., Phillips, N. V., “A Logistics Planning System at W. R. Grace,” Operations Research, Vol. 36, No. 6, 1988, pp. 811-822. doi: $10.1287 / o p r e .36 .6 .811$

[9] Siddiqi, A., de Weck, O., Lee, G., \& Shull, S. "Matrix Modeling Methods for Spaceflight Campaign Logistics Analysis,” Journal of Spacecraft and Rockets, Vol. 46, No. 5, 2009, pp. 1037-1048. doi: $10.2514 / 1.43319$

[10] Grogan P., Armar N., Siddiqi A., de Weck O., Lee G., Jordan E., Shishko R., “A Flexible Architecture and Object-oriented Model for Space Logistics Simulation,” AIAA-2009-6548, AIAA Space 2009 Conference and Exposition, Pasadena, California, September 14-17, 2009.

[11] Ahuja R. K., Magnanti T. L., Orlin J. B., Network Flows, Prentice Hall, 1993.

[12] Jarvis, J. J., and Ratliff H.D., "Some equivalent objectives for dynamic network flow problems,” Management Sci, Vol. 28 No. 1, January 1982, pp. 106-109. doi: 10.1287/mnsc.28.1.106

[13] Silver M., de Weck O., “Time-Expanded Decision Networks: A Framework for Designing Evolvable Complex Systems,” Systems Engineering, Vol. 10, No. 2, 2007, pp. 167-186. doi: 10.1002/sys.20069

[14] Aggarwal C., Ahuja J. H., Hao J., Orlin J. B., “Diagnosing Infeasibilities in Network Flow Problems,” Mathematical Programming, Vol. 81, 1998, pp. 263-280.

[15] Mazanek D. D., Troutman P. A., Culbert C. J., Leonard M. J., Spexarth G., “Surface Buildup Scenarios and Outpost Architectures for Lunar Exploration,” IEEE-1093, 2009 IEEE Aerospace Conference, Big Sky, Montana, March 7-14, 2009.

[16] Kennedy K. J., Toups L. D., Rudisill M., “Constellation Architecture Team - Lunar Scenario 12.0 Habitation Overview,” JSC-CN-19362, Earth and Space 2010 Conference, Honolulu, Hawaii, March 15-17, 2010.

[17] Shull S., Gralla E., de Weck O., Siddiqi A., Shishko R., “The Future of Asset Management for Human Space Exploration,” AIAA-2006-7232, AIAA Space 2006 Conference and Exposition, San Jose, California, Sept. 19-21, 2006. 